\title{
Dynamic Window-Constrained Scheduling for Multimedia Applications *
}

\author{
Richard West and Karsten Schwan \\ College of Computing \\ Georgia Institute of Technology \\ Atlanta, GA 30332
}

\begin{abstract}
This paper describes an algorithm, called Dynamic Window-Constrained Scheduling (DWCS), designed to meet the service constraints on packets from multiple, network-bound media streams with different performance objectives. Using only two attributes, a deadline and a loss-tolerance per packet stream, DWCS: (1) can limit the number of late packets over finite numbers of consecutive packets in loss-tolerant or delayconstrained, heterogeneous traffic streams, (2) does not require a-priori knowledge of the worst-case loading from multiple streams to establish the necessary bandwidth allocations to meet per-stream delay and lossconstraints, and (3) can exhibit both fairness and unfairness properties when necessary. In fact, DWCS can perform fair-bandwidth allocation, static priority $(S P)$ and earliest-deadline first (EDF) scheduling. We show the effectiveness of DWCS using a streaming video application, running over ATM.
\end{abstract}

\section{Introduction}

This paper describes a novel packet scheduling algorithm, called Dynamic Window-Constrained Scheduling (DWCS), that resides at the base of an end-system quality of service (QoS) architecture. DWCS is designed to maximize network bandwidth usage in the presence of multiple packets each with their own delay constraints and loss-tolerances. The algorithm requires two attributes per packet stream, as follows:

- Deadline - this is the latest time a packet can commence service. The deadline is determined from a specification of the maximum allowable time between servicing consecutive packets in the same stream.

- Loss-tolerance - this is specified as a value $x_{i} / y_{i}$, where $x_{i}$ is the number of packets that can be

* This work is supported in part by DARPA through the Honeywell Technology Center under contract numbers B09332478 and B09333218, and by the British Engineering and Physical Sciences Research Council with grant number 92600699. lost or transmitted late for every window, $y_{i}$, of consecutive packet arrivals in the same stream, $i$. Hence, for every $y_{i}$ packet arrivals in stream $i$, a minimum of $y_{i}-x_{i}$ packets must be scheduled for service by their deadlines.

At any time, all packets in the same stream have the same loss-tolerance, while each successive packet in a stream has a deadline that is offset by a fixed amount from its predecessor.

DWCS has the ability to share bandwidth among competing clients in strict proportion to their deadlines and loss-tolerances. This is similar to (weighted) fair scheduling[1, 2, 3, 4], which attempts to allocate bandwidth in proportion to stream weights. Similar proportional share algorithms have been targeted at CPU scheduling $[5,6]$. However, the idea of "windowing' in DWCS is closer to the work of Hamdaoui and Ramanathan[7] who have simulated an algorithm that services multiple streams, in an attempt to ensure at least $m$ customers (packets or threads) in a stream (or process) meet their deadlines for every $k$ consecutive customers from the same stream (or process). In comparison, DWCS can also perform static priority and earliest-deadline first scheduling, supporting both deadline and non-deadline constrained traffic. Furthermore, DWCS can meet explicit delay and 'windowed' loss constraints, using only two attributes that enable a diverse range of service specifications. We now discuss the DWCS algorithm in more detail.

\section{The DWCS Algorithm}

Dynamic Window-Constrained Scheduling (DWCS) orders packets for transmission based on the current values of their loss-tolerances and deadlines. Precedence is given to the packet at the head of the stream with the lowest loss-tolerance. Packets in the same stream all have the same original and current losstolerances, and are scheduled in their order of arrival. Whenever a packet misses its deadline, the losstolerance for all packets in the same stream, $s$, is re- 
duced to reflect the increased importance of transmitting a packet from $s$. This approach avoids starving the service granted to a given packet stream, and attempts to increase the importance of servicing any packet in a stream likely to violate its original loss constraints. Conversely, any packet serviced before its deadline causes the loss-tolerance of other packets (yet to be serviced) in the same stream to be increased, thereby reducing their priority.

\begin{tabular}{||c||}
\hline \multicolumn{1}{||c||}{ Pairwise Packet Ordering } \\
\hline Lowest loss-tolerance first \\
\hline Same non-zero loss-tolerance, order EDF \\
\hline $\begin{array}{c}\text { Same non-zero loss-tolerance \& deadlines, } \\
\text { order lowest loss-numerator first }\end{array}$ \\
\hline Zero loss-tolerance \& denominators, \\
order EDF \\
\hline $\begin{array}{c}\text { Zero loss-tolerance, order } \\
\text { highest loss-denominator first }\end{array}$ \\
\hline All other cases: first-come-first-serve \\
\hline
\end{tabular}

Table 1: Precedence amongst pairs of packets

Table 1 shows the rules for ordering pairs of packets in different streams. Observe that, if two packets have the same non-zero loss-tolerance and deadline they are ordered lowest loss-numerator $x_{i}$ first, where $x_{i} / y_{i}$ is the current loss-tolerance for all packets in stream $i$. By ordering on the lowest loss-numerator, precedence is given to the packet in the stream with tighter loss constraints, since fewer consecutive packet losses can be tolerated. If two packets have zero loss-tolerance and their loss-denominators are both zero, they are ordered EDF, otherwise they are ordered highest lossdenominator first. In such a circumstance, it is possible that a stream may lose more packets than its loss-tolerance specification allows. By increasing the denominator in this case, the algorithm attempts to favor the adversely affected packet stream, bringing the amortized loss for packets in that stream back to the original loss-tolerant value.

Every time a packet in stream $i$ is transmitted, the loss-tolerance of $i$ is adjusted. Likewise, other streams' loss-tolerances are adjusted only if any of the packets in those streams miss their deadlines as a result of queueing delay.

For streams that can lose packets, any packets in these streams that have missed their deadlines are simply discarded. For a stream that cannot lose packets, the deadline serves to minimize queueing delay before eventual transmission of all packets in that stream. The loss-tolerance value for such streams serves to avoid transmitting too many late packets.

We now describe how loss-tolerances are adjusted.
Let $x_{i} / y_{i}$ denote the original loss-tolerance for all packets in stream $i$. Let $x_{i}^{\prime} / y_{i}^{\prime}$ denote the current losstolerance for all queued packets in stream $i$. Let $x_{i}^{\prime}$ denote the current loss-numerator, while $x_{i}$ is the original loss-numerator for packets in stream $i . y_{i}^{\prime}$ and $y_{i}$ denote current and original loss-denominators, respectively. Before a packet stream is serviced, its current and original loss-tolerances are equal. For all buffered packets in the same stream $i$ as the packet most recently transmitted before its deadline, adjust the loss numerators and denominators as follows:

$$
\begin{aligned}
& \text { if }\left(y_{i}^{\prime}>x_{i}^{\prime}\right) \text { then } y_{i}^{\prime}=y_{i}^{\prime}-1 ; \\
& \text { if }\left(x_{i}^{\prime}=y_{i}^{\prime}=0\right) \text { then } x_{i}^{\prime}=x_{i} ; y_{i}^{\prime}=y_{i} \text {; }
\end{aligned}
$$

Observe that loss-tolerances do not change for streams without deadlines. However, for all buffered packets, if any packet in stream $j \mid j \neq i$ misses its deadline:

$$
\begin{aligned}
& \text { if }\left(x_{j}^{\prime}>0\right) \text { then } \\
& \quad x_{j}^{\prime}=x_{j}^{\prime}-1 ; y_{j}^{\prime}=y_{j}^{\prime}-1 ; \\
& \quad \text { if }\left(x_{j}^{\prime}=y_{j}^{\prime}=0\right) \text { then } x_{j}^{\prime}=x_{j} ; y_{j}^{\prime}=y_{j} \text {; } \\
& \text { else if }\left(x_{j}^{\prime}=0\right) \text { then } \\
& \quad \text { if }\left(x_{j}>0\right) \text { then } y_{j}^{\prime}=y_{j}^{\prime}+\left\lceil\frac{y_{j}-x_{j}}{x_{j}}\right\rceil ; \\
& \quad \text { if }\left(x_{j}=0\right) \text { then } y_{j}^{\prime}=y_{j}^{\prime}+y_{j} ;
\end{aligned}
$$

As an example, consider $n=3$ streams of packets, $s_{1}, s_{2}$ and $s_{3}$ (see Figure 1). Let the original loss-tolerances of each stream be $1 / 2,3 / 4$ and $6 / 8$, respectively. Let the deadlines of the first packets in each stream be 0 and let each successive packet $p$ in stream $i$, have a deadline one time unit later than predecessor $(p-1)$ in the same stream $i$. That is, deadline $_{1_{i}}=0$ and deadline $p_{p_{i}}=$ deadline $_{(p-1)_{i}}+1$, $\forall i, 1 \leq i \leq n, p \in Z^{+}$, where $Z^{+}$is the set of positive integers. Assume that the service time of each packet is one time unit. If each stream has a packet arrive for service once every time unit, the total load on the scheduler is 3.0 from all three streams. However, due to the loss-tolerances of each stream, the minimum demand from all streams is $\sum_{i=1}^{n} \frac{\left(1-l_{i}\right) C_{i}}{T_{i}}$, where $l_{i}$ is the loss-tolerance, $C_{i}$ is the service time (or transmission delay) of each and every packet in stream $i$, and $T_{i}$ is the inter-arrival time for packets in stream $i$ (which is also the time between successive packet deadlines). For this example, with the three streams having the above loss-tolerances, the effective scheduler load can be as low as 1.0 if we carefully discard (or service late) appropriate late packets from each stream. Thus, it may still be possible to service all three streams while meeting the appropriate losses from each stream.

From Figure 1, the first packet to be scheduled in this case will be from $s_{1}$, because $s_{1}$ has the lowest loss-tolerance. Since the serviced packet does not miss its deadline, the new (current) loss-tolerance of $s_{1}$ will be set to $1 / 1$. As a result, we can still allow the loss of 
the next packet in $s_{1}$ and not violate the original loss tolerance. Hence, the rationale for adjusting the losstolerance in this way. At time $t=1$, the first packet in $s_{1}$ has been serviced but the first packets in $s_{2}$ and $s_{3}$ have each missed their deadlines. As a result, the first packet in each of these streams is dropped and the new loss-tolerances for $s_{2}$ and $s_{3}$ are set to $2 / 3$ and $5 / 7$, respectively. This change in loss-tolerance compensates for one less allowable packet loss over a range of one fewer packets than in the original losstolerance specification. At time $t=1$, the packet at the head of $s_{2}$ with deadline $=1$ has the highest priority, so it is serviced next. This causes the packets with deadline $=1$ from $s_{1}$ and $s_{3}$ to miss their deadlines. Observe that $s_{1}$ 's loss-tolerance is set back to its original value at this point, because it was temporarily set to $0 / 0$, which is meaningless. Notice that at time $t=5$, a packet in $s_{2}$ gets serviced. When two packets have the same non-zero loss-tolerance and deadline, and their loss-numerators are the same, ties can be broken arbitrarily. Every four time units, the schedule repeats itself. Observe that over the first eight packets serviced, $s_{1}$ transmits four packets and loses four, consuming $50 \%$ of the bandwidth, and both $s_{2}$ and $s_{3}$ transmit two packets and lose six, each consuming $25 \%$ of the bandwidth. Furthermore, one packet from $s_{1}$ is serviced every two time units (or packet service times), one packet from $s_{2}$ is serviced every four time units, and two packets from $s_{3}$ are serviced every eight time units. Hence, the loss-tolerances from all three streams are met.

\begin{tabular}{|c|c|c|c|c|c|c|c|c|c|c|c|c|c|c|c|c|c|}
\hline $\mathrm{s}_{1}$ & $\mathrm{~s}_{2}$ & $\mathrm{~s}_{1}$ & $\mathrm{~s}_{3}$ & $\mathrm{~s}_{1}$ & $\mathrm{~s}_{2}$ & $\mathrm{~s}_{1}$ & $\mathrm{~s}_{3}$ & $\mathrm{~s}_{1}$ & $\mathrm{~s}_{2}$ & $\mathrm{~s}_{1}$ & & \begin{tabular}{l|l}
$\mathrm{s}_{3}$ & $\mathrm{~s}$ \\
\end{tabular} & $\mathrm{~s}_{1}$ & $\mathrm{~s}_{2} \mathrm{~s}$ & $\mathrm{~s}_{1}$ & $\mathrm{~s}_{3}$ & \\
\hline & & & & & & & & & & & & & & & & & \\
\hline & T & $T$ & $T$ & $T$ & $T$ & $T$ & $T$ & T & T & $T$ & $T$ & 1 & $T$ & 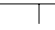 & $T$ & & time, $\mathrm{t}$ \\
\hline 0 & 1 & 2 & 3 & 5 & 6 & 7 & 8 & 8 & 9 & 10 & 11 & $1 \quad 12$ & 213 & $13 \quad 14$ & $4 \quad 15$ & $\begin{array}{ll}5 & 16\end{array}$ & $\longrightarrow$ \\
\hline 1 & & $/ 2(0)$, & ), 1/1 & (1), $1 /$ & $1 / 2(2)$ & 2), $1 / 1$ & 1(3), &, $1 / 2($ & & & & & & & & & \\
\hline So & & $/ 4(0)$ & ),2/3( & (1),2/2 & /2(2), & ), $1 / 1($ & (3),3 & $3 / 4(4$ & & & & $2 / 2(6$ & (6), $1 /$ & $/ 1(7)$ & ),3/4( & (8)... & \\
\hline $\mathrm{s}_{3}$ & & $/ 8(0)$ & ),5/7( & (1),4/6 & $/ 6(2)$, &, $3 / 5$ & (3),3 & & & & & $1 / 2(6$ & (6),, $0 /$ & $/ 1(7)$ & & & \\
\hline
\end{tabular}

Figure 1: Example DWCS scheduling of 3 streams, $s_{1}$, $s_{2}$ and $s_{3}$. Deadlines are shown in brackets and losstolerances are shown as $\mathrm{x} / \mathrm{y}$.

We now describe some features that show the flexibility of DWCS.

Earliest-Deadline First Scheduling using DWCS

When the loss-tolerances of all packets in each stream are set to 0/0, packets are scheduled in EDF order. Intuitively, this makes sense, since all streams have the same importance so their corresponding packets are serviced based upon the time remaining to their deadlines. It can be shown that if all deadlines can be met, EDF guarantees to meet all deadlines. If packets are dropped after missing their deadlines, EDF is optimal with respect to loss-rate in discrete-time G/D/1 and continuous-time $\mathrm{M} / \mathrm{D} / 1$ queues[8].

\section{Static Priority Scheduling using DWCS}

If no packets in any streams have deadlines (ie., they effectively have infinite deadlines), DWCS degrades to static priority (SP). Static-priority scheduling is optimal for a weighted mean delay objective, where weighted mean delay is a linear combination of the delays experienced by all packets[9]. In DWCS, the current loss-tolerances associated with each packet in every stream are always equal to their original losstolerances, and each packet's loss-tolerance serves as its static priority. Namely, for packets with infinite deadlines, the term "loss-tolerance" is really a misnomer, since no packets are actually lost. As expected, precedence is given to the packet with the lowest "losstolerance" (i.e., highest priority). For packets with infinite deadlines, DWCS has the ability to service non-time-constrained packets in static priority order to minimize weighted mean delay.

\section{Fair Scheduling using DWCS}

Fair Queueing derivatives such as SFQ[4] have the ability to share bandwidth among $n$ message streams such that each stream receives a weighted fair-share of available bandwidth. Specifically, let $w_{i}$ be the weight of message stream $i$ and $B_{i}\left(t_{1}, t_{2}\right)$ be the aggregate service (in bits) of $i$ in the interval $\left[t_{1}, t_{2}\right]$. If we consider two message streams, $i$ and $j$, the normalized service (by weight) received by each stream will be $\frac{B_{i}\left(t_{1}, t_{2}\right)}{w_{i}}$ and $\frac{B_{j}\left(t_{1}, t_{2}\right)}{w_{j}}$, respectively. The aim is to ensure that $\left|\frac{B_{i}\left(t_{1}, t_{2}\right)}{w_{i}}-\frac{B_{j}\left(t_{1}, t_{2}\right)}{w_{j}}\right|$ is as close to zero as possible, considering that packets are indivisible entities and an integer number of packets might not be serviced during the interval $\left[t_{1}, t_{2}\right]$.

DWCS also has the ability to meet weighted fair allocation of bandwidth, as shown in Figure 1. Given stream weights, $w_{i}$, in a fair bandwidth-allocating algorithm, we can calculate the loss-tolerances and deadlines that must be assigned to streams in DWCS to give the equivalent bandwidth allocations. This is done as follows:

1. Determine the minimum time window, $\Delta_{\min }$, over which bandwidth is shared proportionally among $n$ streams, each with weight $w_{i} \mid 1 \leq i \leq n, w_{i} \in$ $Z^{+}$: First, let $C_{i}$ be the service time of each packet in stream $i$. (This assumes all packets in any one stream are the same length). Let $\omega=\sum_{i=1}^{n} w_{i}$ and let $\eta_{i}$ be the number of packets from stream $i$ serviced in some arbitrary time window $\Delta$. (Note that $\eta_{i} C_{i}$ is the total service time of stream $i$ over the interval $\Delta$, and $\sum_{i=1}^{n} \eta_{i} C_{i}=\Delta$. Furthermore, 
$\Delta$ is assumed sufficiently large to ensure bandwidth allocations amongst all $n$ streams in exact proportions to their weights). This implies that $\frac{\eta_{i} C_{i}}{\Delta}=\frac{w_{i}}{\omega}$. If $w_{i}$ is a factor of $\omega C_{i}$, let $\gamma_{i}=\frac{\omega C_{i}}{w_{i}}$, else let $\gamma_{i}=\omega C_{i}$. Then $\Delta_{\min }=\operatorname{lcm}\left(\gamma_{1}, \ldots, \gamma_{n}\right)$, where $\operatorname{lcm}(a, b)$ is the lowest-common-multiple of $a$ and $b$.

2. For DWCS, set deadline $1_{i}=0$, and deadline $p_{i}=$ deadline $_{(p-1)_{i}}+C_{i}$, for each packet $p_{i}$ in stream $i$, where $p \in Z^{+}$.

3. To calculate the loss-tolerance, $l_{i}$, of packets in stream $i$, let $l_{i}=\frac{x_{i}}{y_{i}}$, where: $y_{i}=\frac{\Delta_{\text {min }}}{C_{i}}, x_{i}=$ $\frac{\Delta_{\min }}{C_{i}}-\eta_{i}^{\prime}$, and $\eta_{i}^{\prime}=\frac{\eta_{i} \Delta_{\min }}{\Delta}=\frac{w_{i} \Delta_{\min }}{\omega C_{i}}$.

If deadlines are assigned as in step 2, we can translate packet loss-tolerances back into stream weights, $w_{i}$, as follows: $w_{i}=\frac{y_{n}\left(y_{i}-x_{i}\right)}{y_{i}\left(y_{n}-x_{n}\right)}$, where $0<\frac{x_{i}}{y_{i}}<1$.

\section{Experimental Evaluation}

All experiments were performed on a cluster of SparcStation Ultra II Model 2148s. Streams of MPEG1 video frames are placed into shared memory queues, ready for packetization and scheduling. At any point in time there are between one and $n$ active streams $s_{i} \mid 1 \leq i \leq n$, each with their own service attributes.

\section{Fair-Bandwidth Allocation}

The first experiment compares DWCS to SFQ in its ability to achieve fair-bandwidth allocation (link sharing) amongst $n$ streams in the shortest time-frame possible. Weights are assigned to the streams in SFQ, and the corresponding loss-tolerances and deadlines, for DWCS, are computed using the method in Section 2.

Four streams requiring service, comprise bursts of 150 frames with an average arrival rate of 30 frames per second, followed by idle periods with a mean interburst gap of 1 second. The burst periods average 5 seconds and these bursts are repeated 10 times per stream, for a total of 1500 frame (or packet) arrivals per stream. All packets are eventually transmitted, even if they are late. The scheduler interval is $40 \mathrm{mS}$, so 25 frames can be serviced in 1 second. For these experiments, the packet service times are assumed to be equal to the scheduler period, since the scheduler services at most one packetized frame each time it executes. This scenario overloads the scheduler and forces a build-up of back-logged arrivals.

Figure 2 shows the bandwidths (bit service rates) of the four streams over a 50 second period, for SFQ and DWCS. The weights for streams $s_{1}, \ldots, s_{4}$ are $1,1,2$ and 4 , respectively. The corresponding loss-tolerances are $7 / 8,14 / 16,6 / 8$ and $4 / 8$. In the steady-state, SFQ and DWCS behave almost identically, each servicing $s_{1}$ and $s_{2}$ at about $110 \mathrm{Kbps}, s_{3}$ at about $220 \mathrm{Kbps}$, and $s_{4}$ at about $440 \mathrm{Kbps}$.

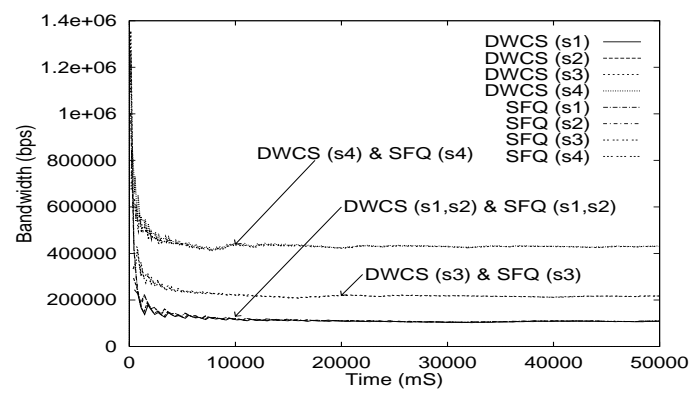

Figure 2: Bandwidth allocations for 4 streams, $s_{1}, \ldots, s_{4}$, with weights, $1,1,2$ and 4 , respectively. The corresponding loss-tolerances are $7 / 8,14 / 16,6 / 8$ and $4 / 8$.

By minimizing the window of time over which bandwidth is allocated in proportion to weights of streams in SFQ, SFQ actually manages to meet the losstolerances and deadlines of packets almost as successfully as DWCS. However, DWCS explicitly uses deadlines and loss-tolerances for packet streams. Hence, bandwidth can be allocated to streams to meet these constraints, independent of the constraints on other streams, assuming enough bandwidth is available, which is the case when $\sum_{i=1}^{n} \frac{\left(1-l_{i}\right) C_{i}}{T_{i}} \leq 1.0$. Note that in the experiments above, at no time did DWCS violate the loss-tolerances on any of its streams even though the scheduler was overloaded and had to service some packets late.

\section{Out-of-Band Traffic}

Consider the scenario where stream $s_{1}$ requires twice as much bandwidth as $s_{2}$ when both streams are active. However, suppose stream $s_{3}$ is carrying timecritical (out-of-band) traffic that must be delivered to its destination with the shortest possible delay. In this case, we always want to grant service to $s_{3}$ when $s_{3}$ has packets for service, but when $s_{3}$ is not active, the bandwidth must be shared between $s_{1}$ and $s_{2}$.

Fair-scheduling algorithms cannot handle the above scenario but DWCS can, because for the duration of a burst of packets from $s_{3}$, that burst must be serviced exclusively. This violates the fairness properties of fair schedulers. In contrast, with DWCS, by carefully choosing a loss-tolerance that reflects the highest priority for $s_{3}$, and by setting the packet deadlines to infinity, DWCS gives exclusive service to $s_{3}$ when it is active. However, as $s_{1}$ and $s_{2}$ are starved of service, their loss-tolerances dynamically decrease, thereby raising their priorities until it is possible that they have precedence over $s_{3}$. The loss-tolerance of 

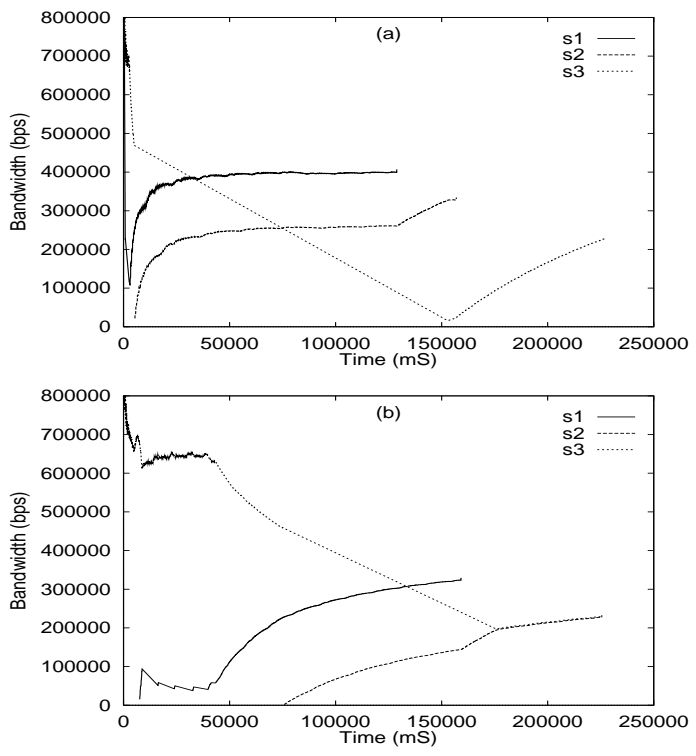

Figure 3: Bandwidth versus time for two dynamic priority streams, $s_{1}$ and $s_{2}$, and a static priority stream, $s_{3}$. (a) Loss-tolerances for $s_{1}, s_{2}$ and $s_{3}$ are $1 / 3,2 / 3$ and $0 / 100$, respectively. (b) Loss-tolerances for $s_{1}, s_{2}$ and $s_{3}$ are $1 / 3,2 / 3$ and $0 / 1500$, respectively.

$s_{3}$ traffic must be set so that the maximum burst size from $s_{3}$ does not require a longer uninterrupted service duration than the time to raise the priority of either $s_{1}$ or $s_{2}$ above that of $s_{3}$.

Figure $3(\mathrm{a})$ shows the results of servicing 3 streams, in which the first two, $s_{1}$ and $s_{2}$, are assigned dynamic priorities (ie., loss-tolerances of $1 / 3$ and $2 / 3$, respectively) that reflect their bandwidth shares, while $s_{3}$ is assigned a static priority of $0 / 100$. Initially, $s_{3}$ receives a greater service rate than either $s_{1}$ or $s_{2}$, but as $s_{1}$ and $s_{2}$ are neglected, their loss-tolerances decrease.

If the loss-tolerance for $s_{3}$ is reduced even further, by increasing its denominator, larger durations of service time are granted to consecutive packet arrivals from $s_{3}$ (as shown in Figure 3(b) where the loss-tolerance of $s_{3}$ is $0 / 1500)$. Observe that $0 / y_{1}$ is higher priority than $0 / y_{2}$ if $y_{1}>y_{2}$. This is one of the precedence rules described in Table 1. Hence, we can fine-tune stream attributes, so that the loss-tolerance of out-of-band data reflects the time to service the largest traffic burst without interruption. Observe that in Figures 3(a) and (b), when the bandwidth curve for $s_{3}$ decreases, it is actually not being serviced. Meanwhile, $s_{1}$ and $s_{2}$ approach their steady-states, close to $2: 1$ bandwidth shares.

\section{Conclusions}

DWCS has the ability to limit the number of late packets over finite numbers of consecutive packets in loss-tolerant or delay-constrained, heterogeneous traf- fic streams. DWCS can support a combination of static priority and dynamic priority (bandwidth-allocated) traffic. In fact, DWCS can perform fair-bandwidth allocation, static priority (SP) and earliest-deadline first (EDF) scheduling. However, unlike fair-scheduling algorithms, DWCS can be unfair when necessary, as well as showing all the fairness characteristics of fairschedulers such as SFQ. By being unfair when necessary, DWCS can schedule out-of-band data in the presence of other loss-tolerant and delay-constrained traffic.

DWCS can meet explicit delay and 'windowed' loss constraints, using only two attributes that enable a diverse range of service specifications. Further details of the algorithm are described in an accompanying paper[10].

\section{References}

[1] A. Demers, S. Keshav, and S. Shenker, "Analysis and simulation of a fair queueing algorithm," Journal of Internetworking Research and Experience, pp. 3-26, October 1990.

[2] S. Golestani, "A self-clocked fair queueing scheme for broadband applications," in INFOCOMM'94, pp. 636646, IEEE, April 1994.

[3] J. C. Bennett and H. Zhang, " $W F^{2} Q$ : Worst-case fair weighted fair queueing," in IEEE INFOCOMM'96, pp. 120-128, IEEE, March 1996.

[4] P. Goyal, H. Vin, and H. Cheng, "Start-time fair queueing: A scheduling algorithm for integrated services packet switching networks," in IEEE SIGCOMM'96, IEEE, 1996.

[5] I. Stoica, H. Abdel-Wahab, K. Jeffay, S. K. Baruah, J. E. Gehrke, and C. G. Plaxton, "A proportional share resource allocation algorithm for real-time, timeshared systems," in Real-Time Systems Symposium, IEEE, December 1996.

[6] C. A. Waldspurger and W. E. Weihl, "Stride scheduling: Deterministic proportional-share resource management," Tech. Rep. MIT/LCS/TM-528, MIT, 1995.

[7] M. Hamdaoui and P. Ramanathan, "A dynamic priority assignment technique for streams with (m,k)-firm deadlines," IEEE Transactions on Computers, April 1995.

[8] S. S. Panwar, D. Towsley, and J. K. Wolf, "Optimal scheduling policies for a class of queues with customer deadlines to the beginning of service," Journal of the $A C M$, vol. 35, pp. 832-844, October 1988.

[9] J. M. Harrison, "Dynamic scheduling of a multiclass queue: Discount optimality," Operations Research, vol. 23, pp. 370-382, March-April 1975.

[10] R. West and K. Schwan, "Dynamic windowconstrained scheduling for multimedia applications," Tech. Rep. GIT-CC-98-18, Georgia Institute of Technology, 1998. 